\section{Local Mode Dependent Decentralized Stabilization of Uncertain Markovian Jump Large-Scale Systems}

\author{
Junlin Xiong, Valery A. Ugrinovskii, and Ian R. Petersen
}

\begin{abstract}
This technical note is concerned with the robust stabilization of a class of stochastic large-scale systems governed by a finite state Markov process. Using the method of integral quadratic constraints, a sufficient condition is developed to design decentralized stabilizing controllers which use local system states and local system operation modes to produce local control inputs. The condition is given in terms of a set of rank constrained linear matrix inequalities. The theory is illustrated by an example.
\end{abstract}

Index Terms-Large-scale systems, linear matrix inequalities (LMIs), Markov jump parameters, stabilization.

\section{INTRODUCTION}

Dynamic systems with high dimensions and complex structures are often referred to as large-scale systems. Many practical examples of such systems are found in fields such as flexible communications networks, economics, and power systems. For the large-scale systems that can be represented as a set of interconnected subsystems, decentralized control has proved to be a useful control technique [1]. Unlike centralized controllers, decentralized controllers only use locally available information of the subsystems. However, the design of decentralized controllers is a challenging task because the dynamics of subsystems are also affected by other subsystems [2].

In this technical note, we consider the stabilization problem for a class of uncertain large-scale systems whose parameters take random values in a finite set; each element of this set determines a particular mode of the system operation [3]. In general, these random events may have complex statistics, however in many applications, a Markov process model provides an acceptable description for system parameter changes [3]. This class of systems, known as Markovian jump systems, has been extensively studied in the control literature; e.g., see [4] and the references therein. In particular, decentralized control of Markovian jump large-scale systems was considered in [5], [6]. A common assumption employed in these and many other papers in this field is that the global operation mode of the system must be known to every controller. That is, while access to the system dynamical states is restricted to the local subsystem states only, the decentralized controllers must be able to access information outside the respective subsystem. This outside information is the complete knowledge of the operation modes of all subsystems; the broadcast of this information is required in many existing control designs.

The main focus of this technical note is the development of a control scheme that eliminates the need for broadcasting the global operation mode. As a result, the designed decentralized controllers are fully autonomous; they only use the state and mode information locally available within the subsystems. To emphasize this feature of the proposed control scheme, we refer to this type of controllers as the local mode dependent decentralized controllers. Also, the proposed control

Manuscript received March 04, 2009; revised April 06, 2009 and July 20, 2009. First published October 16, 2009; current version published November 04, 2009. This work was supported by the Australian Research Council. Recommended by Associate Editor J. Lygeros.

The authors are with the School of Engineering and Information Technology, University of New South Wales at the Australian Defence Force Academy, Canberra ACT 2600, Australia (e-mail: junlin.xiong@gmail.com; j.xiong@adfa. edu.au; v.ugrinovskii@gmail.com; i.r.petersen@gmail.com).

Digital Object Identifier 10.1109/TAC.2009.2031565 scheme generally allows us to reduce the number of control gains to be scheduled for each subsystem, compared to the techniques using the global operation mode. This suggests that our solution may be more practical in applications. The controller design procedure proposed in this technical note also takes advantage of the knowledge of the system connectivity structure and its uncertainty structure; this information is embedded into the coupled Riccati equations and the corresponding coupled LMIs that one needs to solve to obtain the control gains.

Another feature of the uncertain large-scale systems studied in this technical note is that the uncertainties in subsystems and the unknown interconnections between subsystems are described in terms of integral quadratic constraints (IQCs) [7]. The class of uncertainties described by an IQC can include nonlinearities, time-varying uncertainties and dynamic uncertainties with bounded $H_{\infty}$ norms [7]. The possibility to allow for dynamic uncertainties is particularly useful; it allows us to account for unmodelled dynamics in the interconnections between subsystems. The IQC uncertainty description has been used in recent results on the robust stabilization of jump large-scale systems using global mode dependent decentralized controllers [5], [6].

This technical note focuses on the decentralized stabilization problem via local mode dependent state feedback. The problem of robust local mode dependent decentralized output feedback control has been considered recently in [8].

The technical note is organized as follows. Section II formulates the class of uncertain large-scale systems governed by a finite-state Markov process. It should be noted that this Markov process is not assumed to be decomposable or nearly decomposable into smaller Markov processes; therefore the systems under consideration cannot be broken into smaller independent or weakly connected Markov jump subsystems. The main results on decentralized stabilization via local mode dependent control are developed in Section III. A numerical example is presented in Section IV to compare our theory with the results of [5]. Section V concludes the technical note.

Notation: $\mathbb{R}^{+}$denotes the set of positive real numbers. $\mathbb{S}^{+}$denotes the set of real symmetric positive definite matrices. The notation $X \geq$ $Y$ (respectively, $X>Y$ ) means that $X-Y$ is positive semi-definite (respectively, positive definite). $\|\cdot\|$ refers to the Euclidean norm for vectors and the induced 2-norm for matrices. Moreover, let $(\Omega, \mathcal{F}, \operatorname{Pr})$ be a complete probability space $\operatorname{Pr}(\cdot)$ is the probability measure, and $\mathrm{E}(\cdot)$ denotes the mathematical expectation operator.

\section{PROBLEM FORMULATION}

Consider an uncertain Markovian jump large-scale system $\mathcal{S}$ consisting of $N$ subsystems $\mathcal{S}_{i}, i \in \mathcal{N} \triangleq\{1,2, \ldots, N\}$. The $i$ th subsystem is described by

$$
\mathcal{S}_{i}:\left\{\begin{array}{r}
\dot{x}_{i}(t)=A_{i}\left(\eta_{i}(t)\right) x_{i}(t)+B_{i}\left(\eta_{i}(t)\right) u_{i}(t) \\
+E_{i}\left(\eta_{i}(t)\right) \xi_{i}(t)+L_{i}\left(\eta_{i}(t)\right) r_{i}(t) \\
\zeta_{i}(t)=H_{i}\left(\eta_{i}(t)\right) x_{i}(t)
\end{array}\right.
$$

where $x_{i}(t) \in \mathbb{R}^{n_{i}}$ is the state of subsystem $\mathcal{S}_{i}, u_{i}(t) \in \mathbb{R}^{m_{i}}$ is the control input, $\xi_{i}(t) \in \mathbb{R}^{p_{i}}$ is the local uncertainty input, $\zeta_{i}(t) \in \mathbb{R}^{q_{i}}$ is the uncertainty output, $r_{i}(t) \in \mathbb{R}^{s_{i}}$ is the interconnection input, which describes the effect of other subsystems $\mathcal{S}_{j}, j \neq i$, on $\mathcal{S}_{i}$ due to the interconnections between subsystem $\mathcal{S}_{i}$ and subsystems $\mathcal{S}_{j}, j \neq$ $i$. The process $\eta_{i}(t)$ denotes the operation mode of subsystem $\mathcal{S}_{i}$; it takes values in a finite state space $\mathcal{M}_{i} \triangleq\left\{1,2, \ldots, M_{i}\right\}$. The initial condition of subsystem $\mathcal{S}_{i}$ is given by $x_{i}(0)=x_{i 0} \in \mathbb{R}^{n_{i}}$ and $\eta_{i}(0)=$ $\eta_{i 0} \in \mathcal{M}_{i}$.

Each local mode process $\eta_{i}(t)$ is assumed to be a stationary random process defined on $(\Omega, \mathcal{F}, \operatorname{Pr})$. These local mode processes are not 
required to be Markov processes, and may depend on each other. The controllers to be designed in this technical note will only be able to access the states of these non-Markov mode processes. However, the collective vector mode process $\left(\eta_{1}(t), \ldots, \eta_{N}(t)\right)$ describing the mechanism of mode changes in the entire large-scale system will be assumed to be a stationary ergodic Markov process. The state space of this vector process is assumed to consist of $M$ distinct vectors. It is generally smaller than the product-space $\mathcal{M}_{1} \times \cdots \times \mathcal{M}_{N}$, i.e., $M \leq \prod_{i=1}^{N} M_{i}$, due to the possibility of dependency between the mode processes of individual subsystems. In the sequel, it will be convenient to use a bijective mapping between the state space of the vector process $\left(\eta_{1}(t), \ldots, \eta_{N}(t)\right)$ and the set $\mathcal{M} \triangleq\{1,2, \ldots, M\}$. This mapping is defined in Section III. It maps the random vector process $\left(\eta_{1}(t), \ldots, \eta_{N}(t)\right)$ into an $\mathcal{M}$-valued random scalar process $\eta(t)$, which will be referred to as the global operation mode process of the entire large-scale system. Therefore, $\eta(t)$ is a stationary ergodic Markov process, and its state transition probability rate matrix is assumed to be $\mathbf{Q}=\left(q_{\mu \nu}\right) \in \mathbb{R}^{M \times M}$, in which $q_{\mu \nu} \geq 0$ if $\nu \neq \mu$, and $q_{\mu \mu} \triangleq-\sum_{\nu=1, \nu \neq \mu}^{M} q_{\mu \nu}$.

The uncertainties and interconnections in the large-scale system are described by operators

$$
\begin{aligned}
& \xi_{i}(t)=\phi_{i}^{\xi}\left(t,\left.\zeta_{i}(\cdot)\right|_{0} ^{t},\left.\eta_{i}(\cdot)\right|_{0} ^{t}\right) \\
& r_{i}(t)=\phi_{i}^{r}\left(t,\left.\underline{\zeta}_{i}(\cdot)\right|_{0} ^{t},\left.\eta(\cdot)\right|_{0} ^{t}\right)
\end{aligned}
$$

where $\underline{\zeta}_{i}(\cdot) \triangleq\left[\zeta_{1}(\cdot) \cdots \zeta_{i-1}(\cdot) \zeta_{i+1}(\cdot) \cdots \zeta_{N}(\cdot)\right]$. They are assumed to satisfy the following IQCs [5], [6], [9].

Definition 1: Given a set of matrices $\bar{S}_{i} \in \mathbb{S}^{+}, i \in \mathcal{N}$. A collection of local uncertainty inputs $\xi_{i}(t), i \in \mathcal{N}$, is an admissible local uncertainty for the large-scale system if there exists a sequence $\left\{t_{l}\right\}_{l=1}^{\infty}$ such that $t_{l} \geq 0, t_{l} \rightarrow \infty$, and for all $l$ and for all $i \in \mathcal{N}$

$$
\mathrm{E}\left(\int_{0}^{t_{l}}\left[\left\|\zeta_{i}(t)\right\|^{2}-\left\|\xi_{i}(t)\right\|^{2}\right] d t \mid x_{0}, \eta_{0}\right) \geq-x_{i 0}^{T} \bar{S}_{i} x_{i 0}
$$

where $x_{0}=\left[x_{10}^{T}, \ldots, x_{N 0}^{T}\right]^{T}$, and $\eta_{0}=\eta(0)$. The set of the admissible local uncertainties is denoted by $\Xi$.

Definition 2: Given a set of matrices $\tilde{S}_{i} \in \mathbb{S}^{+}, i \in \mathcal{N}$. The largescale system is said to have admissible interconnections between subsystems if there exists a sequence $\left\{t_{l}\right\}_{l=1}^{\infty}$ such that $t_{l} \geq 0, t_{l} \rightarrow \infty$, and for all $l$ and for all $i \in \mathcal{N}$, we have

$$
\begin{aligned}
& \mathrm{E}\left(\int_{0}^{t_{l}}\left[\left(\sum_{j=1, j \neq i}^{N}\left\|\zeta_{j}(t)\right\|^{2}\right)-\left\|r_{i}(t)\right\|^{2}\right] d t \mid x_{0}, \eta_{0}\right) \\
& \geq-x_{i 0}^{T} \tilde{S}_{i} x_{i 0} .
\end{aligned}
$$

The set of the admissible interconnections is denoted by $\Pi$. Without loss of generality, we assume that the same sequence $\left\{t_{l}\right\}_{l=1}^{\infty}$ is used in both definitions.

Remark 1: The IQCs on the uncertainties and interconnections in the above definitions capture a broad class of nonlinearities, time-varying, static and dynamic uncertainties and interconnection signals. Indeed, norm-bounded uncertainties of the form $\xi_{i}(t)=\Delta_{i}(t) \zeta_{i}(t)$ satisfying the condition $\left\|\Delta_{i}(t)\right\| \leq 1$, also satisfy the IQC (2) with arbitrary $\bar{S}_{i}>0$ and $\left\{t_{l}\right\}_{l=1}^{\infty}$. Hence such uncertainties belong to $\Xi$. Also, exogenous $L_{2}$-integrable disturbances $\xi_{i}(t)=w_{i}(t)$ such that $\int_{0}^{\infty}\left\|w_{i}(t)\right\|^{2} d t \leq x_{i 0}^{T} \bar{S}_{i} x_{i 0}$ satisfy the IQC (2). Furthermore, dynamic uncertain interconnections represented in operator form as $r_{i}(s)=\Delta_{i}(s) \zeta_{i}(s)$, where $\Delta_{i}(s)$ is a transfer function from the Hardy space $R H^{\infty}$ such that $\left\|\underline{\Delta}_{i}(s)\right\|_{\infty} \leq 1$, also satisfy the IQC (3) for arbitrary $\tilde{S}_{i}>0$ and $\left\{t_{l}\right\}_{l=1}^{\infty}$ [7]. Hence, such interconnections belong to $\Pi$.
Given the system (1) subject to the uncertainty constraints (2), (3), the objective of this technical note is to design a local mode dependent decentralized state-feedback controller of the form

$$
u_{i}(t)=K_{i}\left(\eta_{i}(t)\right) x_{i}(t)
$$

such that the resulting closed-loop system is robustly stochastically stable in the following sense.

Definition 3: The closed-loop system corresponding to the uncertain system (1)-(3) and the controller (4) is said to be robustly stochastically stable if there exists a constant $c_{1} \in \mathbb{R}^{+}$such that $x_{i}(\cdot) \in \mathbb{L}_{2}[0, \infty)$ for all $i \in \mathcal{N}$, and

$$
\sum_{i=1}^{N} \mathrm{E}\left(\int_{0}^{\infty}\left\|x_{i}(t)\right\|^{2} d t \mid x_{0}, \eta_{0}\right) \leq c_{1} \sum_{i=1}^{N}\left\|x_{i 0}\right\|^{2}
$$

for any initial conditions $x_{0}$ and $\eta_{0}$, any admissible local uncertainty $\xi_{i}(t)$ and any admissible interconnection $r_{i}(t), i \in \mathcal{N}$.

Remark 2: The robust stochastic stability of the closed-loop largescale system defined above is equivalent to the absolute stability of the closed-loop system considered in [5] and [6].

Remark 3: The operation mode process in the controller (4) is the local mode process $\eta_{i}(t)$, whereas the controllers considered in [5] and [6] are dependent on the global mode process $\eta(t)$. It is worth emphasizing that the requirement of knowing $\eta(t)$ in [5] and [6] is equivalent to the requirement of knowing the operation mode information of all the subsystems. On the other hand, the local mode processes $\eta_{i}(t)$ are, in general, not Markovian even when the global mode process $\eta(t)$ is Markovian. This generally rules out the possibility of designing a local mode dependent stabilizing controller by considering each subsystem in isolation. Unfortunately, the majority of the existing control design tools assume the Markov nature of the mode process.

Remark 4: As a result of the local mode dependency of the controller (4), the control scheme proposed in this technical note promises several operational advantages. Firstly, the gains of the decentralized controllers are functions of the local operation modes of subsystems. This eliminates the need for broadcasting the global operation mode throughout the entire system. Secondly, our controllers change their gains only when the corresponding subsystems change their operation modes. In contrast, the global mode dependent controllers studied in [5] and [6] change their gains whenever any of the subsystems switches to a different mode. This often results in undesirable transient dynamics in the subsystems. Thirdly, the hardware implementation of a local mode dependent control scheme is simpler since in general, a fewer number of control gains need to be scheduled. Indeed, if controller (4) is used, we need to implement $M_{i}$ control gains for the subsystem $\mathcal{S}_{i}$. The total number of the control gains to be implemented will be $\sum_{i=1}^{N} M_{i}$. If one uses global mode dependent controllers such as those proposed in [5], [6], then $M$ control gains are needed for each subsystem. Hence the total number of the control gains will be $N M\left(\geq \sum_{i=1}^{N} M_{i}\right)$. These comments indicate that our control scheme may offer some practical advantages compared to the schemes using the global operation mode.

\section{CONTROller Design}

This section presents the main results of the technical note. To obtain a local mode dependent controller, first in Section III-A we formalize the relationship between the local operation modes of the subsystems and the global operation modes of the entire system. In Section III-B, we show how a local mode dependent controller can be derived from a given global mode dependent controller; the result of that section is a sufficient condition to ensure that such a derivation is possible. To reduce conservatism, our method uses least conservative (for a specified augmented uncertainty class) auxiliary global mode dependent controllers as a starting point. The design of the auxiliary controllers is 
described in Section III-C, where we present a sufficient condition for the existence of such controllers based on the results of [5], [6]. Then, in Section III-D, we propose a local mode dependent controller design technique using the auxiliary global mode dependent controllers presented in Section III-C. In Section III-E, we combine these derivations and formulate them as a feasibility problem for a set of rank constrained LMIs.

\section{A. Operation Modes}

Let $\mathcal{M}_{\mathrm{p}}$ be a non-empty subset of the set $\mathcal{M}_{1} \times \mathcal{M}_{2} \times \cdots \times \mathcal{M}_{N}$, which consists of the states visited by the vector mode process $\left(\eta_{1}(t), \eta_{2}(t), \ldots, \eta_{N}(t)\right)$. Because both $\mathcal{M}_{\mathrm{p}}$ and $\mathcal{M}$ have $M$ elements, a bijection exists between them. We denote such a bijective mapping by $\Psi: \mathcal{M}_{\mathrm{p}} \rightarrow \mathcal{M}$ and write $\mu=\Psi\left(\mu_{1}, \mu_{2}, \ldots, \mu_{N}\right)$ where $\mu \in \mathcal{M}$ and $\mu_{i} \in \mathcal{M}_{i}$. The inverse function $\left(\mu_{1}, \mu_{2}, \ldots, \mu_{N}\right)=$ $\Psi^{-1}(\mu)$ defines the mapping $\Psi^{-1}: \mathcal{M} \rightarrow \mathcal{M}_{\mathrm{p}}$. Also, the components of the inverse function $\Psi^{-1}$ are denoted by $\Psi_{i}^{-1}$, i.e., $\mu_{i}=\Psi_{i}^{-1}(\mu)$; they define the mappings $\Psi_{i}^{-1}: \mathcal{M} \rightarrow \mathcal{M}_{i}$ for each $i \in \mathcal{N}$.

If the operation modes of the subsystems are not constrained, then the number of the global operation modes is $M=\prod_{i=1}^{N} M_{i}$, and $\mathcal{M}_{\mathrm{p}}=\mathcal{M}_{1} \times \mathcal{M}_{2} \times \cdots \times \mathcal{M}_{N}$. However, even in this case, the local mode processes $\eta_{i}(t)$, which are the components of the vector process $\left(\eta_{1}(t), \eta_{2}(t), \ldots, \eta_{N}(t)\right)$, may in general be dependent and may not satisfy the Markov condition. The following example illustrates the relationship among the operation modes when there are mode constraints.

Example 1: Let $N=3, M_{1}=M_{2}=M_{3}=2$. The set $\mathcal{M}_{1} \times \mathcal{M}_{2} \times \mathcal{M}_{3}$ consists of 8 triplets $\left(\mu_{1}, \mu_{2}, \mu_{3}\right), \mu_{i} \in\{1,2\}$; it denotes all possible mode patterns of the subsystems. However, if there are constraints on the operation modes of the subsystems, say $\eta_{2}(t)=\eta_{3}(t)$ if $\eta_{1}(t)=1$, and $\eta_{2}(t) \neq \eta_{3}(t)$ otherwise, then only four mode combinations are possible, and $\mathcal{M}_{\mathrm{p}}=\{(1,1,1),(1,2,2),(2,1,2),(2,2,1)\}$. Hence the large-scale system has 4 global operation modes, i.e., $\mathcal{M}=\{1,2,3,4\}$. In this case, the bijection $\Psi$ can be defined using the correspondence table between $\mathcal{M}_{\mathrm{p}}$ and $\mathcal{M}$. Also, let the state transition probability rate matrix of $\eta(t)$ be

$$
\mathbf{Q}=\left[\begin{array}{cccc}
-0.35 & 0.2 & 0.1 & 0.05 \\
0.1 & -2 & 0.7 & 1.2 \\
0.4 & 0.9 & -1.45 & 0.15 \\
0.1 & 0.2 & 0.3 & -0.6
\end{array}\right]
$$

One can verify by direct calculations that $\eta_{1}(t)$ and $\eta_{2}(t)$ are dependent, and $\eta_{2}(t)$ is not Markov; see [10].

\section{B. Design Methodology}

To study the stabilization of the uncertain system $\mathcal{S}$ consisting of the systems (1) and the uncertainty constraints (2) and (3), we first study an auxiliary class of uncertain systems that contains the uncertain system $\mathcal{S}$ as a special case. The reason we adopt such an approach is that there exist tight necessary and sufficient conditions for the design of decentralized stabilizing controllers for this auxiliary class of uncertain large-scale systems.

Consider a class of uncertain large-scale systems given by

$$
\left\{\begin{aligned}
\dot{\tilde{x}}_{i}(t)= & \tilde{A}_{i}(\eta(t)) \tilde{x}_{i}(t)+\tilde{B}_{i}(\eta(t))\left[\tilde{u}_{i}(t)+\tilde{\xi}_{i}^{u}(t)\right] \\
& +\tilde{E}_{i}(\eta(t)) \tilde{\xi}_{i}(t)+\tilde{L}_{i}(\eta(t)) \tilde{r}_{i}(t) \\
\tilde{\zeta}_{i}(t)= & \tilde{H}_{i}(\eta(t)) \tilde{x}_{i}(t)
\end{aligned}\right.
$$

where $\tilde{A}_{i}(\mu)=A_{i}\left(\mu_{i}\right), \tilde{B}_{i}(\mu)=B_{i}\left(\mu_{i}\right), \tilde{E}_{i}(\mu)=E_{i}\left(\mu_{i}\right), \tilde{L}_{i}(\mu)=$ $L_{i}\left(\mu_{i}\right), \tilde{H}_{i}(\mu)=H_{i}\left(\mu_{i}\right), \mu \in \mathcal{M}$, and $\mu_{i}=\Psi_{i}^{-1}(\mu) \in \mathcal{M}_{i}$. The uncertainty inputs $\tilde{\xi}_{i}(t)$ and $\tilde{r}_{i}(t)$ are, respectively, generated by the same operators as $\xi_{i}(t)$ and $r_{i}(t)$ in (1), and satisfy the IQCs in (2),
(3), where $\zeta_{i}(t), \xi_{i}(t)$ and $r_{i}(t)$ are replaced with $\tilde{\zeta}_{i}(t), \tilde{\xi}_{i}(t)$ and $\tilde{r}_{i}(t)$, respectively. We denote the corresponding admissible uncertainty sets as $\left\{\tilde{\xi}_{i}(t)\right\} \in \tilde{\Xi}$ and $\left\{\tilde{r}_{i}(t)\right\} \in \tilde{\Pi}$. Furthermore, $\tilde{\xi}_{i}^{u}(t)$ is the uncertainty in the control input, and is described by a function of the form $\tilde{\xi}_{i}^{u}(t)=$ $\phi_{i}^{u}\left(t,\left.\tilde{x}_{i}(\cdot)\right|_{0} ^{t},\left.\eta(\cdot)\right|_{0} ^{t}\right)$, which satisfies the following IQC.

Definition 4: Given $\beta_{i}^{u}(\mu) \in \mathbb{R}^{+}, i \in \mathcal{N}, \mu \in \mathcal{M}$. A collection of uncertainty inputs $\tilde{\xi}_{i}^{u}(t), i \in \mathcal{N}$, is an admissible uncertainty input for the auxiliary large-scale system (6) if

$$
\mathrm{E}\left(\int_{0}^{t_{l}}\left(\beta_{i}^{u}(\eta(t))\left\|\tilde{x}_{i}(t)\right\|^{2}-\left\|\tilde{\xi}_{i}^{u}(t)\right\|^{2}\right) d t \mid \tilde{x}_{0}, \eta_{0}\right) \geq 0
$$

for all $l$ and for all $i \in \mathcal{N}$. Here, $\left\{t_{l}\right\}_{l=1}^{\infty}$ is the same sequence as in Definitions 1,2. This set of the admissible uncertainty inputs is denoted by $\tilde{\Xi}^{u}$.

Under IQC uncertainty and interconnection constraints, the design of robust global mode dependent decentralized controllers for the uncertain system (6) has been studied in [5]. In that reference, a global mode dependent decentralized controller of the form

$$
\tilde{u}_{i}(t)=\tilde{K}_{i}(\eta(t)) \tilde{x}_{i}(t)
$$

was obtained such that the closed-loop system consisting of (2), (3), (6)-(8), is robustly stochastically stable and achieves bounded worstcase quadratic performance $\sup _{\tilde{\Xi}, \tilde{\Pi}, \tilde{\Xi} u} J<c, c \in \mathbb{R}^{+}$, measured in terms of the quadratic cost

$$
\begin{aligned}
J \triangleq \sum_{i=1}^{N} \mathrm{E} & \left(\int _ { 0 } ^ { \infty } \left[\tilde{x}_{i}^{T}(t) Q_{i}(\eta(t)) \tilde{x}_{i}(t)\right.\right. \\
& \left.\left.+\tilde{u}_{i}^{T}(t) R_{i}(\eta(t)) \tilde{u}_{i}(t)\right] d t \mid \tilde{x}_{0}, \eta_{0}\right)
\end{aligned}
$$

where $Q_{i}(\mu) \in \mathbb{S}^{+}, R_{i}(\mu) \in \mathbb{S}^{+}, i \in \mathcal{N}, \mu \in \mathcal{M}$. Under some additional assumptions on $\eta(t)$ (see Remark 5), the controller (8) achieves the optimal worst-case performance, and the conditions on its existence are necessary and sufficient; e.g., see [5]. However, the control gain in (8) depends on $\eta(t)$ and is not local mode dependent. The following result relates the robust stabilization of the system (1) via local mode dependent decentralized controller (4) with the robust stabilization of the auxiliary system (6) using the controller (8).

Theorem 1: Suppose controller (8) stochastically stabilizes the uncertain large-scale system (6) subject to the constraints (2), (3), (7). If the control gains $K_{i}(\cdot)$ in (4) are chosen so that

$$
\left\|\tilde{K}_{i}(\mu)-K_{i}\left(\mu_{i}\right)\right\|^{2} \leq \beta_{i}^{u}(\mu)
$$

where $\mu \in \mathcal{M}, \mu_{i}=\Psi_{i}^{-1}(\mu) \in \mathcal{M}_{i}$, for all $i \in \mathcal{N}$, then the controller in (4) stochastically stabilizes the uncertain large-scale system (1) subject to the constraints (2) and (3).

Proof: The idea here follows that in [9] and [11]. Define $\Delta_{i}(\mu) \triangleq$ $\tilde{K}_{i}(\mu)-K_{i}\left(\mu_{i}\right)$. Then (10) implies $\left\|\Delta_{i}(\mu)\right\|^{2} \leq \beta_{i}^{u}(\mu)$. Consider a particular uncertainty input of the form $\tilde{\xi}_{i}^{u}(t)=-\Delta_{i}(\eta(t)) \tilde{x}_{i}(t)$. We have $\left\|\tilde{\xi}_{i}^{u}(t)\right\|^{2} \leq \beta_{i}^{u}(\eta(t))\left\|\tilde{x}_{i}(t)\right\|^{2}$, therefore this uncertainty input satisfies the constraint (7). Hence, the closed-loop system consisting of the auxiliary large-scale system (6) driven by this particular uncertainty input and the controller (8) is robustly stable over $\tilde{\Xi}$ and $\tilde{\Pi}$. Also, we have

$$
\tilde{u}_{i}(t)+\tilde{\xi}_{i}^{u}(t)=\left[\tilde{K}_{i}(\eta(t))-\Delta_{i}(\eta(t))\right] \tilde{x}_{i}(t)=K_{i}\left(\eta_{i}(t)\right) \tilde{x}_{i}(t)
$$


which is of the same form as in (4). Hence, the class of the closed-loop systems modelled by (1)-(4) is a subclass of the class of the closed-loop systems modelled by (2), (3), and (6)-(8). Therefore, the stability of the closed-loop system consisting of the uncertain system (1) and the controller (4), subject to (2), (3), and (10), follows from the stability of the auxiliary closed-loop system established above.

\section{Design of Global Mode Dependent Controllers}

In this section, a sufficient condition is established for the design of an auxiliary stabilizing controller of the form (8). This condition, together with Theorem 1, provides a basis for the design of local mode dependent stabilizing controllers of the form (4).

Theorem 2:

1) If there exist constants $\tau_{i} \in \mathbb{R}^{+}, \theta_{i} \in \mathbb{R}^{+}, \tau_{i}^{u} \in \mathbb{R}^{+}, i \in \mathcal{N}$, such that the coupled algebraic Riccati equations (AREs)

$$
\begin{aligned}
& \tilde{A}_{i}^{T}(\mu) X_{i}(\mu)+X_{i}(\mu) \tilde{A}_{i}(\mu)+\sum_{\nu=1}^{M} q_{\mu \nu} X_{i}(\nu)+Q_{i}(\mu) \\
& +\left[\tau_{i}+\sum_{j=1, j \neq i}^{N} \theta_{j}\right] \tilde{H}_{i}^{T}(\mu) \tilde{H}_{i}(\mu)+\tau_{i}^{u} \beta_{i}^{u}(\mu) I \\
& -X_{i}(\mu)\left[\tilde{B}_{i}(\mu) R_{i}^{-1}(\mu) \tilde{B}_{i}^{T}(\mu)-\frac{1}{\tau_{i}^{u}} \tilde{B}_{i}(\mu) \tilde{B}_{i}^{T}(\mu)\right. \\
& \left.-\frac{1}{\tau_{i}} \tilde{E}_{i}(\mu) \tilde{E}_{i}^{T}(\mu)-\frac{1}{\theta_{i}} \tilde{L}_{i}(\mu) \tilde{L}_{i}^{T}(\mu)\right] X_{i}(\mu)=0
\end{aligned}
$$

have solutions $X_{i}(\mu) \in \mathbb{S}^{+}$for all $i \in \mathcal{N}, \mu \in \mathcal{M}$, then the controller (8) given by

$$
\tilde{K}_{i}(\mu)=-R_{i}^{-1}(\mu) \tilde{B}_{i}^{T}(\mu) X_{i}(\mu)
$$

robustly stabilizes the uncertain system (6) subject to the constraints (2), (3), and (7), and leads to the cost bound $J \leq \sum_{i=1}^{N} x_{i 0}^{T}\left[X_{i}\left(\eta_{i 0}\right)+\tau_{i} \bar{S}_{i}+\theta_{i} \tilde{S}_{i}\right] x_{i 0}$.

2) The claim in 1) remains true if instead of the AREs (11) one uses the coupled algebraic Riccati inequalities obtained by replacing the " =" sign in (11) with " $<$."

Proof: Define a controlled output for the system (6) as

$$
\tilde{z}_{i}(t)=\left[\begin{array}{c}
Q_{i}^{1 / 2}(\eta(t)) \\
0
\end{array}\right] \tilde{x}_{i}(t)+\left[\begin{array}{c}
0 \\
R_{i}^{1 / 2}(\eta(t))
\end{array}\right] \tilde{u}_{i}(t) .
$$

Then the cost functional (9) can be rewritten as $J=$ $\sum_{i=1}^{N} \mathrm{E}\left(\int_{0}^{\infty}\left\|\tilde{z}_{i}(t)\right\|^{2} d t \mid \tilde{x}_{0}, \eta_{0}\right)$. The rest of the proof is similar to the proof of Theorem 1 in [5]. The proof for Part 2 follows the same lines except the LMI version of the bounded real lemma is used.

Remark 5: If, in addition, there exists a transition group of measurepreserving set transformations $\Gamma_{s}: \Omega \rightarrow \Omega$ such that $\eta\left(t, \Gamma_{s} \omega\right)=$ $\eta(t+s, \omega)$ almost surely for all $t, s \geq 0$; e.g., see [12, Ch. XI], then the sufficient condition in Part 1 of Theorem 2 is also necessary, provided the conditional expectations involved in the cost $J$ and its bound, given $\tilde{x}_{0}$ and $\eta_{0}$, are replaced with the conditional expectations given $\tilde{x}_{0}$. This fact follows from the lossless S-procedure result; see Lemma 2 and Theorem 1 in [5]. Therefore, the controller obtained in Theorem 2 is worst-case optimal under these additional assumptions.

Remark 6: To solve the AREs (11) numerically, one may use the iteration methods proposed in [13]. Also, the corresponding coupled algebraic Riccati inequalities can be solved by translating them into coupled LMIs using the Schur complement equivalence.

\section{Design of Local Mode Dependent Controllers}

Suppose a global mode dependent controller (8) is given. In this section we describe how a corresponding local mode dependent controller of the form (4) can be constructed. Also, we give a probabilistic interpretation of the proposed design.

Theorem 3: Given the controller (8). Let

$$
K_{i}\left(v_{i}\right)=\frac{\sum_{\mu=1}^{M}\left\{\tilde{K}_{i}(\mu) \pi_{\infty \mu} \rrbracket_{i}\left(\mu, v_{i}\right)\right\}}{\sum_{\mu=1}^{M}\left\{\pi_{\infty \mu} \rrbracket_{i}\left(\mu, v_{i}\right)\right\}}
$$

for all $v_{i} \in \mathcal{M}_{i}, i \in \mathcal{N}$, where $\rrbracket_{i}\left(\mu, v_{i}\right)=1$ if $v_{i}=\Psi_{i}^{-1}(\mu)$, and $\mathbb{V}_{i}\left(\mu, v_{i}\right)=0$ otherwise, $\pi_{\infty \mu}$ is the $\mu$ th component of the vector $\pi_{\infty}=\mathbf{e}(\mathbf{Q}+\mathbf{E})^{-1}, \mathbf{e}=\left[\begin{array}{llll}1 & 1 & \cdots & 1\end{array}\right] \in \mathbb{R}^{1 \times M}$, and $\mathbf{E}=\left[\begin{array}{llll}\mathbf{e}^{T} & \mathbf{e}^{T} & \cdots & \mathbf{e}^{T}\end{array}\right]^{T} \in \mathbb{R}^{M \times M}$. Then $K_{i}\left(v_{i}\right)=$ $\lim _{t \rightarrow \infty} \mathrm{E}\left(\tilde{K}_{i}(\eta(t)) \mid \eta_{i}(t)=v_{i}\right)$.

Proof: First we observe that the vector $\pi_{\infty}$ is the steady state distribution of the global mode process $\eta(t)$. The ergodic property of the Markov process $\eta(t)$ implies that $\lim _{t \rightarrow \infty} e^{\mathbf{Q} t}=\left[\begin{array}{lll}\pi_{\infty}^{T} & \cdots & \pi_{\infty}^{T}\end{array}\right]^{T}$. Then the probability distribution of the control gain of (8) has a limit as $t \rightarrow \infty$, which is $\lim _{t \rightarrow \infty} \operatorname{Pr}\left(\tilde{K}_{i}(\eta(t))=\tilde{K}_{i}(\mu)\right)=\pi_{\infty \mu}$ for $\mu \in \mathcal{M}$. So the expected value of the global mode dependent control gain conditioned on the subsystem operation mode, as $t \rightarrow \infty$, is given by

$$
\begin{aligned}
\lim _{t \rightarrow \infty} & \mathrm{E}\left(\tilde{K}_{i}(\eta(t)) \mid \eta_{i}(t)=v_{i}\right) \\
= & \sum_{\mu=1}^{M}\left\{\tilde{K}_{i}(\mu) \lim _{t \rightarrow \infty} \operatorname{Pr}\left(\eta(t)=\mu \mid \eta_{i}(t)=v_{i}\right)\right\} \\
= & \sum_{\mu=1}^{M}\left\{\tilde{K}_{i}(\mu) \lim _{t \rightarrow \infty} \frac{\operatorname{Pr}\left(\eta(t)=\mu, \eta_{i}(t)=v_{i}\right)}{\operatorname{Pr}\left(\eta_{i}(t)=v_{i}\right)}\right\} \\
= & \sum_{\mu=1}^{M}\left\{\tilde{K}_{i}(\mu) \frac{\pi_{\infty \mu} \rrbracket_{i}\left(\mu, v_{i}\right)}{\sum_{\mu=1}^{M}\left\{\pi_{\infty \mu} \rrbracket_{i}\left(\mu, v_{i}\right)\right\}}\right\} \\
= & K_{i}\left(v_{i}\right) .
\end{aligned}
$$

This completes the proof.

Note that by choosing the controller (13) to be the limit of the conditional expectation of (8) given the subsystem operation mode, we introduce the asymptotically most accurate local mode dependent approximation of the given global mode dependent controller. Indeed, the control gain in (13) is the minimum variance approximation of the control gain in (8), in the sense that $\lim _{t \rightarrow \infty} \mathrm{E}\left(\left\|\tilde{K}_{i}(\eta(t))-K_{i}\left(\eta_{i}(t)\right)\right\|_{\mathrm{F}}^{2} \mid \eta_{i}(t)\right)$, is minimal $[14$, Theor. 3.1]; here $\|\cdot\|_{\mathrm{F}}$ denotes the Frobenius norm.

It immediately follows from (13) that

$$
\tilde{K}_{i}(\mu)-K_{i}\left(\mu_{i}\right)=\frac{\sum_{\nu=1, \nu \neq \mu}^{M}\left\{\rrbracket_{i}\left(\nu, \mu_{i}\right) \pi_{\infty \nu}\left[\tilde{K}_{i}(\mu)-\tilde{K}_{i}(\nu)\right]\right\}}{\sum_{\nu=1}^{M}\left\{\rrbracket_{i}\left(\nu, \mu_{i}\right) \pi_{\infty \nu}\right\}}
$$

for all $\mu \in \mathcal{M}, \mu_{i}=\Psi_{i}^{-1}(\mu) \in \mathcal{M}_{i}$. Note that the expression on the right-hand side of the above equation is a linear function of $\tilde{K}_{i}(\cdot)$. In the next section, this will allow us to derive a bound on $\left\|\tilde{K}_{i}(\mu)-K_{i}\left(\mu_{i}\right)\right\|$ from an LMI involving parameters of $\tilde{K}_{i}(\cdot)$.

\section{E. Computational Method}

A computational method for the design of the controller (4) is presented in this subsection. The method is based upon Theorems 1, 2, 
and 3, and is formulated as a feasibility problem for a set of rank constrained LMIs.

For all $i \in \mathcal{N}, \mu \in \mathcal{M}$, consider the coupled LMIs

$$
\begin{gathered}
{\left[\begin{array}{lcc}
\Upsilon_{i 11}(\mu) & \Upsilon_{i 12}(\mu) & \Upsilon_{i 13}(\mu) \\
\Upsilon_{i 12}^{T}(\mu) & \Upsilon_{i 22}(\mu) & 0 \\
\Upsilon_{i 13}^{T}(\mu) & 0 & \Upsilon_{i 33}(\mu)
\end{array}\right]<0} \\
{\left[\begin{array}{cc}
\bar{\tau}_{i}^{u} I & \Delta_{i}(\mu) \\
\Delta_{i}^{T}(\mu) & \tilde{\beta}_{i}(\mu) I
\end{array}\right] \geq 0} \\
{\left[\begin{array}{cc}
\bar{\beta}_{i}(\mu) & 1 \\
1 & \tilde{\beta}_{i}(\mu)
\end{array}\right] \geq 0, \quad\left[\begin{array}{cc}
\bar{X}_{i}(\mu) & I \\
I & X_{i}(\mu)
\end{array}\right] \geq 0}
\end{gathered}
$$

with rank constraints

$\operatorname{rank}\left(\left[\begin{array}{cc}\bar{\beta}_{i}(\mu) & 1 \\ 1 & \tilde{\beta}_{i}(\mu)\end{array}\right]\right) \leq 1, \operatorname{rank}\left(\left[\begin{array}{cc}\bar{X}_{i}(\mu) & I \\ I & X_{i}(\mu)\end{array}\right]\right) \leq n$

where we get

$$
\begin{aligned}
& \Upsilon_{i 11}(\mu)=\bar{X}_{i}(\mu) \tilde{A}_{i}^{T}(\mu)+\tilde{A}_{i}(\mu) \bar{X}_{i}(\mu)+q_{\mu \mu} \bar{X}_{i}(\mu) \\
& \text { - } \tilde{B}_{i}(\mu) R_{i}^{-1}(\mu) \tilde{B}_{i}^{T}(\mu)+\bar{\tau}_{i}^{u} \tilde{B}_{i}(\mu) \tilde{B}_{i}^{T}(\mu) \\
& +\bar{\tau}_{i} \tilde{E}_{i}(\mu) \tilde{E}_{i}^{T}(\mu)+\bar{\theta}_{i} \tilde{L}_{i}(\mu) \tilde{L}_{i}^{T}(\mu) \\
& \Upsilon_{i 12}(\mu)=\bar{X}_{i}(\mu)\left[\begin{array}{lllll}
I & I & \tilde{H}_{i}^{T}(\mu) & \cdots & \tilde{H}_{i}^{T}(\mu)
\end{array}\right] \\
& \Upsilon_{i 22}(\mu)=-\operatorname{diag}\left(Q_{i}^{-1}(\mu), \bar{\beta}_{i}(\mu) I, \bar{\tau}_{i} I, \bar{\theta}_{1} I, \ldots, \bar{\theta}_{i-1} I,\right. \\
& \left.\bar{\theta}_{i+1} I, \ldots, \bar{\theta}_{N} I\right) \\
& \Upsilon_{i 13}(\mu)=\bar{X}_{i}(\mu)\left[\sqrt{q_{\mu, 1}} I \cdots \sqrt{q_{\mu, \mu-1}} I\right. \\
& \left.\sqrt{q_{\mu, \mu+1}} I \cdots \sqrt{q_{\mu, M}} I\right] \\
& \Upsilon_{i 33}(\mu)=-\operatorname{diag}\left(\bar{X}_{i}(1), \ldots, \bar{X}_{i}(\mu-1),\right. \\
& \left.\bar{X}_{i}(\mu+1), \ldots, \bar{X}_{i}(M)\right) \\
& \Delta_{i}(\mu)=\frac{1}{\sum_{\nu=1}^{M}\left\{\rrbracket_{i}\left(\nu, \mu_{i}\right) \pi_{\infty \nu}\right\}} \sum_{\nu=1, \nu \neq \mu}^{M}\left\{\rrbracket_{i}\left(\nu, \mu_{i}\right) \pi_{\infty \nu}\right. \\
& \left.\times\left[R_{i}^{-1}(\nu) \tilde{B}_{i}^{T}(\nu) X_{i}(\nu)-R_{i}^{-1}(\mu) \tilde{B}_{i}^{T}(\mu) X_{i}(\mu)\right]\right\}
\end{aligned}
$$

and $\mu_{i}$ in $\Delta_{i}(\mu)$ is given by $\mu_{i}=\Psi_{i}^{-1}(\mu)$.

Theorem 4: Suppose there exist matrices $X_{i}(\mu) \in \mathbb{S}^{+}, \bar{X}_{i}(\mu) \in$ $\mathbb{S}^{+}$, scalars $\tilde{\beta}_{i}(\mu) \in \mathbb{R}^{+}, \bar{\beta}_{i}(\mu) \in \mathbb{R}^{+}, \bar{\tau}_{i}^{u} \in \mathbb{R}^{+}, \bar{\tau}_{i} \in \mathbb{R}^{+}, \bar{\theta}_{i} \in \mathbb{R}^{+}$, $i \in \mathcal{N}, \mu \in \mathcal{M}$, such that the matrix inequalities (14)-(17) are satisfied. Using the solution matrices $X_{i}(\mu)$, construct the control gains in (12), and then using (13), construct the controller (4). Then the obtained controller (4) is a stabilizing controller for the uncertain system (1) subject to the constraints (2) and (3).

Proof: Using the Schur complement equivalence, the LMI (14) is equivalent to

$$
\begin{aligned}
& \bar{X}_{i}(\mu) \tilde{A}_{i}^{T}(\mu)+\tilde{A}_{i}(\mu) \bar{X}_{i}(\mu)+q_{\mu \mu} \bar{X}_{i}(\mu)+\bar{\tau}_{i}^{u} \tilde{B}_{i}(\mu) \tilde{B}_{i}^{T}(\mu) \\
& +\sum_{\nu=1, \nu \neq \mu}^{M} q_{\mu \nu} \bar{X}_{i}(\mu)\left(\bar{X}_{i}(\nu)\right)^{-1} \bar{X}_{i}(\mu)-\tilde{B}_{i}(\mu) R_{i}^{-1}(\mu) \tilde{B}_{i}^{T}(\mu) \\
& +\bar{X}_{i}(\mu)\left[Q_{i}(\mu)+\bar{\beta}_{i}^{-1}(\mu) I+\left[\bar{\tau}_{i}^{-1}+\sum_{j=1, j \neq i}^{N} \bar{\theta}_{j}^{-1}\right]\right. \\
& \left.\times \tilde{H}_{i}^{T}(\mu) \tilde{H}_{i}(\mu)\right] \bar{X}_{i}(\mu) \\
& +\bar{\tau}_{i} \tilde{E}_{i}(\mu) \tilde{E}_{i}^{T}(\mu)+\bar{\theta}_{i} \tilde{L}_{i}(\mu) \tilde{L}_{i}^{T}(\mu)<0
\end{aligned}
$$

Furthermore, the LMIs in (16) with rank constraints (17) are equivalent to the constraints

$$
\bar{X}_{i}(\mu)=\left(X_{i}(\mu)\right)^{-1}, \quad \bar{\beta}_{i}(\mu)=\left(\tilde{\beta}_{i}(\mu)\right)^{-1} .
$$

Let $\tau_{i}=\bar{\tau}_{i}^{-1}, \theta_{i}=\bar{\theta}_{i}^{-1}, \tau_{i}^{u}=\left(\bar{\tau}_{i}^{u}\right)^{-1}, \beta_{i}^{u}(\mu)=\bar{\beta}_{i}^{-1}(\mu) \bar{\tau}_{i}^{u}$. Then by pre- and post-multiplying both sides of (18) by $X_{i}(\mu)$, we establish that the conditions in Part 2 of Theorem 2 are satisfied. Therefore, the global mode dependent controller (8) can be constructed using (12), which stabilizes the corresponding auxiliary system (6) subject to the constraints (2), (3), and (7).

Also, it follows from the LMI (15) and the equation (12) that $\left\|\tilde{K}_{i}(\mu)-K_{i}\left(\mu_{i}\right)\right\| \|^{2} \leq \beta_{i}^{u}(\mu)$. Therefore, from Theorem 1 , the constructed controller (4) is stabilizing.

Remark 7: Due to the rank constraints (17), the solution set to (14)-(17) is non-convex. In general, it is difficult to solve such problems. Fortunately, several numerical algorithms have been proposed for this purpose, such as the cone complementarity linearization algorithm in [15], the tangent and lift method in [16].

\section{AN Illustrative EXAMPLE}

We consider a large-scale system of the form (1) which consists of three subsystems; each subsystem has two modes. The modes are governed by the Markov process introduced in Example 1. The system coefficients were chosen randomly in $[-1,1]$ and are given in the supplement [10].

To illustrate the developed methodology, we compare the performance of our local mode dependent controller with that of the global mode dependent controller studied in [5], in terms of the cost (9). To this end, instead of solving the feasibility problem stated in Theorem 4, the local mode dependent controller was designed by solving the optimization problem $\min \gamma$ subject to the conditions in Theorem 4 and the additional condition $\sum_{i=1}^{N} x_{i 0}^{T}\left[\sum_{\mu=1}^{M} \pi_{\infty \mu} X_{i}(\mu)+\tau_{i} \bar{S}_{i}+\theta_{i} \tilde{S}_{i}\right] x_{i 0}<\gamma$. The gains of the resulting local mode dependent controller (4) can also be found in [10]. The controller consists of six gains, two for each subsystem. With the designed controller, the upper bound on the quadratic cost (9) was evaluated for the closed-loop system by solving the corresponding worst-case performance analysis problem derived from [5]; this bound was found to be 8.9677 . For comparison, the exact value of optimal worst-case performance achievable in this system using global mode dependent control is 5.5804; this value was found using [5, Theor. 2]. Note that using [5] leads to a 4-gain schedule for each subsystem and 12 control gains in total. Although the local mode dependent controller did not achieve the same level of performance as the optimal controller, such a performance is achieved with half the number of control gains and without the knowledge of the operation modes of other subsystems. Simulation results demonstrating the performance of the proposed controller are given in [10].

\section{Conclusions}

This technical note has studied the decentralized state feedback stabilization problem for a class of uncertain Markovian jump large-scale systems. The proposed controllers are entirely decentralized with respect to the subsystems. That is, they use local system states and local operation modes of the subsystems to produce local control inputs. A sufficient condition in terms of rank constrained linear matrix inequalities has been developed to construct such controllers. The developed theory has been illustrated by a numerical example.

\section{REFERENCES}

[1] N. R. Sandell, P. Varaiya, M. Athans, and M. G. Safonov, "Survey of decentralized control methods for large scale systems," IEEE Trans. Automat. Control, vol. AC-23, no. 2, pp. 108-128, Apr. 1978.

[2] W.-J. Wang and Y. H. Chen, "Decentralized robust control design with insufficient number of controllers," Int. J. Control, vol. 65, no. 6, pp. 1015-1030, 1996. 
[3] M. Mariton, Jump Linear Systems in Automatic Control. New York: Marcel Dekker, 1990.

[4] O. L. V. Costa, M. D. Fragoso, and R. P. Marques, Discrete-Time Markov Jump Linear Systems. Berlin, Germany: Springer-Verlag, 2005.

[5] V. A. Ugrinovskii and H. R. Pota, "Decentralized control of power systems via robust control of uncertain Markov jump parameter systems," Int. J. Control, vol. 78, no. 9, pp. 662-677, 2005.

[6] L. Li, V. A. Ugrinovskii, and R. Orsi, "Decentralized robust control of uncertain Markov jump parameter systems via output feedback," Automatica, vol. 43, no. 11, pp. 1932-1944, 2007.

[7] I. R. Petersen, V. A. Ugrinovskii, and A. V. Savkin, Robust Control Design Using $H_{\infty}$ Methods. Berlin, Germany: Springer-Verlag, 2000.

[8] J. Xiong, V. A. Ugrinovskii, and I. R. Petersen, "Local mode dependent output feedback control of uncertain Markovian jump large-scale systems," Proc. American Control Conf., pp. 4254-4259, 2009.

[9] I. R. Petersen, "Decentralized state feedback guaranteed cost control of uncertain systems with uncertainty described by integral quadratic constraints," Proc. American Control Conf., pp. 1333-1339, 2006.

[10] J. Xiong, V. A. Ugrinovskii, and I. R. Petersen, Illustrative Example: System Data, Controllers and Simulation Results 2009 [Online]. Available: http://www.ee.adfa.edu.au/staff/valu/XiUP1-supplement.pdf

[11] L. Li and I. R. Petersen, "A rank constrained LMI algorithm for decentralized state feedback guaranteed cost control of uncertain systems with uncertainty described by integral quadratic constraints," Proc. American Control Conf., pp. 796-801, 2007.

[12] J. L. Doob, Stochastic Processes. New York: Wiley, 1953.

[13] H. Abou-Kandil, G. Freiling, and G. Jank, "Solution and asymptotic behavior of coupled Riccati equations in jump linear systems," IEEE Trans. Automat. Control, vol. 39, no. 8, pp. 1631-1636, Aug. 1994.

[14] B. D. O. Anderson and J. B. Moore, Optimal Filtering. Upper Saddle River, NJ: Prentice-Hall, 1979.

[15] L. El Ghaoui, F. Oustry, and M. A. Rami, "A cone complementarity linearization algorithm for static output-feedback and related problems," IEEE Trans. Automat. Control, vol. 42, no. 8, pp. 1171-1176, Aug. 1997.

[16] R. Orsi, U. Helmke, and J. B. Moore, "A Newton-like method for solving rank constrained linear matrix inequalities," Automatica, vol. 42, no. 11 , pp. $1875-1882$, Nov. 2006.

\section{Partially-Linear Least-Squares Regularized Regression for System Identification}

\author{
Yong-Li Xu and Di-Rong Chen
}

\begin{abstract}
In this technical note, we propose a partially-linear least-squares regularized regression (PL-LSRR) method for system identification. This method identifies a general nonlinear function as a sum of two functions which come from a linear and a nonlinear function space respectively. Both the linear and nonlinear functions can involve all regressors. Therefore, the PL-LSRR can make use of the partially-linear structure of a given system to reduce prediction errors more efficiently than exiting partially-linear identification methods. Two examples show that the PL-LSRR can reduce prediction errors and estimate the true linear expansion of the system well.
\end{abstract}

Index Terms-Learning theory, least-squares regularized regression, partially-linear model, reproducing kernel Hilbert space, system identification.

\section{INTRODUCTION}

System identification is an important tool in both science and engineering. Knowing only a set of signal samples $\{(u(t), y(t))\}_{t=1}^{m}$, our goal is to estimate a relation between an input $u(t)$ and an output $y(t)$ and to use this relation to evaluate on new samples. Let $z(t)$ be the regression vector in an ARX model, $z(t)=[y(t-1) ; \ldots ; y(t-$ $p) ; u(t) ; u(t-1) ; \ldots ; u(t-q)]$. Suppose that a system can be formulated as $y(t)=g(z(t))+e(t)$, where $e(t)$ is a stochastic disturbance term with mean 0 . The objective of identification is to find a good approximant $f$ to $g$. Specially, if $g(z(t))=\beta^{\top} z(t)$, the system is a linear system, where $\beta$ is a coefficient vector. Otherwise, the system is a nonlinear system. Many problems related to linear system identification have been solved theoretically. Nonlinear systems are more complex and have received increasing attention. There are different techniques to solve nonlinear black-box identification problems, such as artificial neural networks [8], support vector machines (SVM) [2], [11], [12], wavelets [15], basis expansions [10], splines [13], and polynomials [9].

For some nonlinear systems the partially-linear structure can be exploited by identification algorithms. Let us consider the system in [5] $y(t)=0.5 y(t-1)^{3}-0.5 y(t-2) u(t)+0.3 u(t)-0.2 u(t-1)+$ $e(t)$. Intuitively, $+0.3 u(t)-0.2 u(t-1)$ is its linear component and $0.5 y(t-1)^{3}-0.5 y(t-2) u(t)$ is its nonlinear component. If the system is identified as a full linear system, it will have large prediction errors because the nonlinear component can not be well identified. However, if the system is treated as a full nonlinear system, the linear component is identified as a part of nonlinear component. In this case, a full nonlinear identification method may have better performance than a full linear identification method, but it may be more complex. Partially-linear identification methods may reduce the complexity and prediction errors [5], [6].

Manuscript received March 02, 2009; revised July 05, 2009. First published October 21, 2009; current version published November 04, 2009. This work was supported in part by the National Science Foundation of China under Grant 10871015 and National Basic Research Program of China under Grants 9732010CB731900 and 973-2006CB303102. Recommended by Associate Editor J.-F. Zhang.

The authors are with the Department of Mathematics and LMIB, Beijing University of Aeronautics and Astronautics, Beijing 100091, China (e-mail: xuyongli2312@sina.com; drchen@buaa.edu.cn).

Color versions of one or more of the figures in this technical note are available online at http://ieeexplore.ieee.org.

Digital Object Identifier 10.1109/TAC.2009.2031566 